\title{
Unraveling the Intrinsic and Robust Nature of van Hove Singularities in Twisted Bilayer Graphene by Scanning Tunneling Microscopy and Theoretical Analysis
}

\author{
I. Brihuega,${ }^{1}$ P. Mallet,${ }^{2}$ H. González-Herrero, ${ }^{1}$ G. Trambly de Laissardière,${ }^{3}$ M. M. Ugeda, ${ }^{1}$ L. Magaud, ${ }^{2}$ \\ J. M. Gómez-Rodríguez, ${ }^{1}$ F. Ynduráin, ${ }^{1}$ and J.-Y. Veuillen ${ }^{2, *}$ \\ ${ }^{1}$ Departamento Física de la Materia Condensada, Universidad Autónoma de Madrid, E-28049 Madrid, Spain \\ ${ }^{2}$ Institut Néel, CNRS-UJF, BP 166, F-38042 Grenoble, France \\ ${ }^{3}$ Laboratoire de Physique Théorique et Modélisation, Université de Cergy-Pontoise-CNRS, F-95302 Cergy-Pontoise, France
}

(Received 13 July 2012; published 8 November 2012; publisher error corrected 9 November 2012)

\begin{abstract}
Extensive scanning tunneling microscopy and spectroscopy experiments complemented by firstprinciples and parametrized tight binding calculations provide a clear answer to the existence, origin, and robustness of van Hove singularities (vHs) in twisted graphene layers. Our results are conclusive: vHs due to interlayer coupling are ubiquitously present in a broad range (from $1^{\circ}$ to $10^{\circ}$ ) of rotation angles in our graphene on $6 \mathrm{H}$ - $\mathrm{SiC}(000-1)$ samples. From the variation of the energy separation of the $\mathrm{vHs}$ with the rotation angle we are able to recover the Fermi velocity of a graphene monolayer as well as the strength of the interlayer interaction. The robustness of the vHs is assessed both by experiments, which show that they survive in the presence of a third graphene layer, and by calculations, which test the role of the periodic modulation and absolute value of the interlayer distance. Finally, we clarify the role of the layer topographic corrugation and of electronic effects in the apparent moiré contrast measured on the STM images.
\end{abstract}

Soon after the discovery of the unique electronic properties of graphene [1-3], suggestions were made for engineering the band structure of this material. It has been proposed that periodic potentials with wavelengths in the nanometer range could lead to anisotropic renormalization of the velocity of low energy charge carriers [4] or to the generation of new massless Dirac fermions [5]. Experimental works intended for verifying these theoretical predictions were recently reported [6-8], where the periodic perturbation was generated either by a lattice mismatch with the supporting material or by a self-organized array of clusters. An alternative route for modifying graphene's band structure would be to exploit a rotation between stacked graphene layers [9]. According to calculations, for large angles $\left(\theta \geq 15^{\circ}\right)$ the low energy band structure of graphene should be preserved [10-12]. For intermediate angles $\left(1^{\circ} \leq \theta \leq 15^{\circ}\right)$, it is predicted that, while the linear dispersion persists in the vicinity of the Dirac points of both layers, the band velocity is depressed and two saddle points appear in the band structure, giving rise to two logarithmic van Hove singularities $(\mathrm{vHs})$ in the density of states (DOS) [9,13-18]. For smaller angles $\left(\theta \leq 1^{\circ}\right)$ weakly dispersive bands appear at low energy $[19,20]$ with sharp DOS peaks very close to the Dirac point $[17,18]$.

Twisted graphene layers are commonly found on different substrates, such as metals $[13,21,22]$, the $\mathrm{C}$ face of $\mathrm{SiC}$ [23-25], or graphite surfaces [26,27]. Transfer techniques yielding large domains of twisted bilayers over a macroscopic sample [28] and quantitative, fast, Raman characterization tools $[29,30]$ have recently been proposed. However, despite the fact that rotated graphene layers are readily available and a number of measurements have confirmed that large twist angles lead to an electronic decoupling of stacked graphene layers [3,11,24,31-34], few experiments tackle the electronic properties for sufficiently small angles $\left(\theta<15^{\circ}\right)[13,35,36]$. In particular, recent scanning tunneling microscopy and spectroscopy (STM and STS) studies have demonstrated the renormalization of the band velocity [35] and the appearance of van Hove singularities in the local DOS (LDOS) [13] of three twisted layers configurations, one measured at the graphite surface and two on few layers graphene (FLG) grown on $\mathrm{Ni}$. At variance, a careful angle resolved photoemission spectroscopy investigation of FLG on the $\mathrm{SiC} \mathrm{C}$ face detected neither a van Hove singularity nor any significant change in the Fermi velocity in the range $1^{\circ}<\theta<10^{\circ}$, and suggests major problems in our current understanding of twisted graphene layers [36].

Although twisted bilayers with small rotation angles (typically $\theta \leq 10^{\circ}$ ) appear as a fascinating field of development for the physics of graphene [22,37,38], uncertainties about the mechanism of interlayer interaction remain $[36,39]$. Here we show that graphene layers rotated between $1^{\circ}$ and $10^{\circ}$ present singularities in the LDOS. Our numerical simulations confirm that they arise from a partial gap opening at the crossing points of Dirac cones from neighboring layers, and correspond to logarithmic vHs generated by the interlayer coupling. The vHs are found to be robust against deviations from the ideal twisted bilayer model both in the simulations and in the experiments on the real samples. We find that, as a result of the interlayer rotation, the graphene layers are, in addition, corrugated. The nature and effect of such corrugation in the $\mathrm{vHs}$ has been understood by a 
quantitative comparison with density functional theory (DFT) calculations.

As experimental realization of twisted layers we have chosen a 5 layers thick graphene grown in ultrahigh vacuum on a $6 H$-SiC $(000-1)$ substrate following the procedure described in Ref. [23]. STM images [40] reveal coherent domains with lateral size around $100 \mathrm{~nm}$. Within these domains, the rotations between the surface graphene layers give rise to superstructures with period $P$ in the nanometer range, which are identified as moiré patterns (MP) [23,25-27]. The STM or STS experiments have been made in a separate UHV system using a homemade low temperature (5 K) STM [41]. Conductance spectra were taken using a lock-in technique, with an ac voltage (frequency, $830 \mathrm{~Hz}$; amplitude, $1-10 \mathrm{mV} \mathrm{rms}$ ) added to the dc sample bias. The sample was perfectly regenerated in situ by high temperature annealing $[42,43]$. The data were analyzed using the WSXM software [44].

To settle the origin of the structures seen in STS data we have performed band structure and DOS calculations for twisted bilayers using two different approaches. The first one is a first-principles DFT calculation based on the SIESTA code [45], including van der Waals interactions. This allows testing the roles of the atomic relaxation and corrugation, the interlayer distance, the importance of the van der Waals forces, etc. [40]. The second one is a tight binding (TB) approach [14], which allows handling very large supercells (the implementation of the TB scheme is detailed in Ref. [17]).

The main experimental results of this report are summarized in Fig. 1. Figure 1(c) shows STM images of four single period (simple) MP corresponding to rotation angles ranging from $9.6^{\circ}$ to $1.4^{\circ}$. The rotation angle $\theta$ between neighboring carbon planes can be obtained from the value of the moiré period $P$, using $\sin (\theta / 2)=0.123 / P(\mathrm{~nm})$ $[23,27]$, and the drawing in Fig. 1(a) illustrates the MP for a $9.6^{\circ}$ rotation angle. As depicted in Fig. 1(b), the Brillouin zones of the graphene layers are equally rotated by $\theta$. Thus, the Dirac cones of each layer are now centered in different points of the reciprocal space $K_{1}$ and $K_{2}$. The cones merge into two saddle points at energies $\pm E_{\mathrm{vHs}}$ from the Dirac point, leading to vHs which generate peaks in the DOS. LDOS spectra taken on the MP in Fig. 1(c) are shown in Fig. 1(d). Each spectrum displays two peaks, indicated by arrows, identified as vHs by our theoretical calculations. They are approximately symmetric with respect to the Fermi level $E_{F}$ and evolve towards lower bias for decreasing rotation angle. We have measured the energy separation of the $\mathrm{vHs}$ as a function of the rotation angle $\theta$ for a large number $(\approx 30)$ of simple MP and the result is shown as filled circles in Fig. 1(e). We also plot as crosses the only three other data that had been reported to date, measured for small rotation angles $\left(\theta<4^{\circ}\right)$ in different substrates, which all fall at compatible energies [13]. Finally, as quoted previously [13], our spectra show that

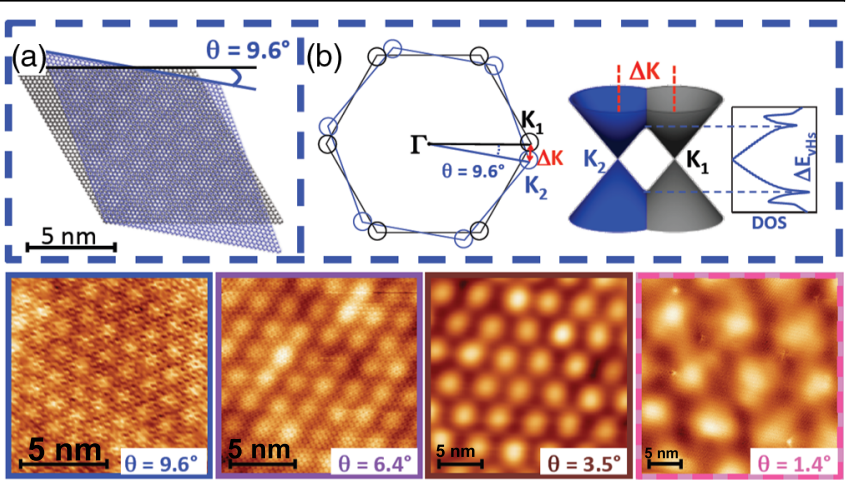

(c)
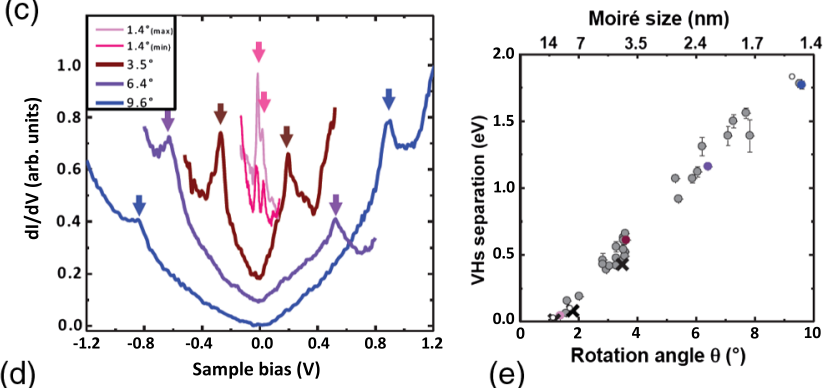

(e)

FIG. 1 (color online). (a) Illustration of a MP arising from a rotation angle $\theta=9.6^{\circ}$. (b) Emergence of vHs as a consequence of the rotation in reciprocal space. (c) STM images of several MP with different $\theta$. The scale bar is $5.0 \mathrm{~nm}$. (d) LDOS spectra taken on the MP shown in (c). The curves are shifted vertically for clarity. The arrows point to the vHs. For $\theta=1.4^{\circ}, \max (\min )$ indicates a spectrum taken on a bright (dark) area. (e) vHs separation as a function of $\theta$. Crosses: Data from Ref. [13]. Filled and open circles: This work. Colored dots refer to the spectra displayed in (d) (same color code). Open circles correspond to multiple MP discussed in Fig. 3.

for small angles $\left(\theta<3.5^{\circ}\right)$ the peaks are localized in the bright regions of the MP, see the upper two spectra in Fig. 1(d) for $\theta=1.4^{\circ}$, a localization which disappears for larger angles, where vHs are completely homogenous across the MP $[13,40]$.

The DOS of twisted bilayers computed for rotation angles $1^{\circ}<\theta<10^{\circ}$ are shown in Fig. 2. As in our experimental data, both ab initio [Fig. 2(a)] and TB [Fig. 2(b)] approaches show two main peaks, which shift towards larger energy with increasing angle. Band structure calculations $[14,17,40]$ show that these peaks are associated with the avoided crossing of the bands of the two layers along the line connecting $K_{1}$ and $K_{2}$, as illustrated in Fig. 1(b). The calculations thus demonstrate that the peaks correspond to the vHs singularity described for smaller rotation angles [13]. The TB calculations of the LDOS in Fig. 2(b) show that the vHs tend to localize in the regions of the MP with $A A$ stacking at small rotation angle [14,17] in a simple one electron picture. This is again in agreement with experimental data [Fig. 1(d) and Ref. [13]], since these regions correspond to the bright areas in the STM images of the MPs, as shown below. 

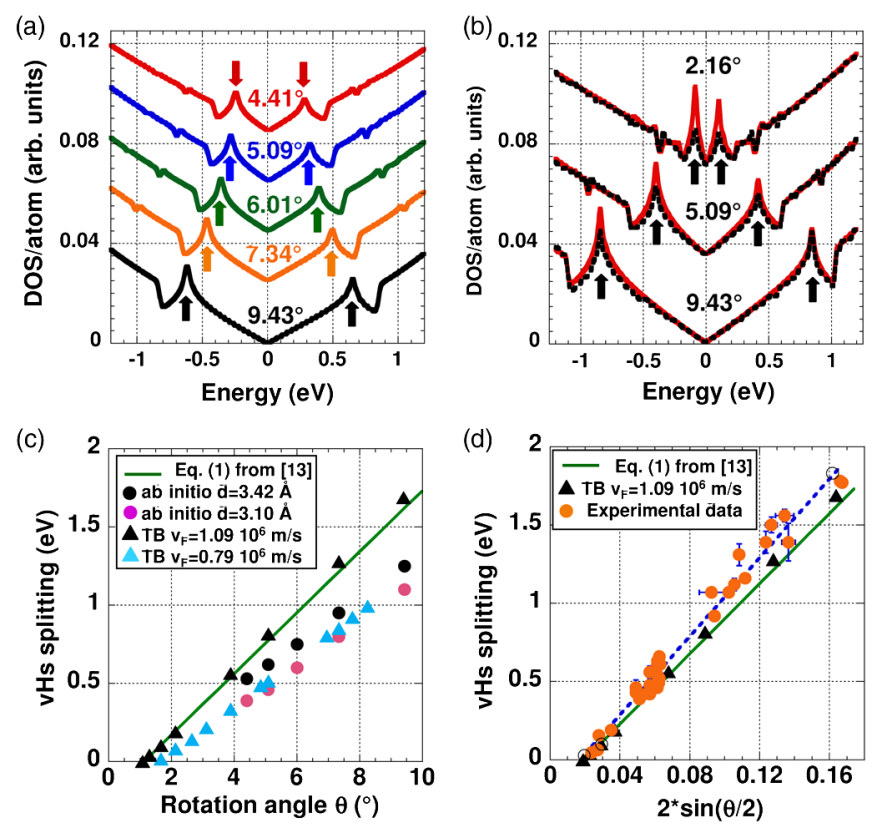

FIG. 2 (color online). (a) $A b$ initio calculations of the total DOS, interlayer distance $d=3.42 \AA$. (b) DOS calculations using the TB formalism with $v_{F}=1.1 \times 10^{6} \mathrm{~m} / \mathrm{s}$. The red continuous line is the LDOS in $A A$ stacked areas, the black dotted line is the total DOS. The spectra have been shifted for clarity. (c) Computed vHs splitting as a function of $\theta$. Green line: Eq. (1) with $v_{F}=1.0 \times 10^{6} \mathrm{~m} / \mathrm{s}$ and $t_{\theta}=0.11 \mathrm{eV}$ [13], black [gray (pink)] circles: ab initio calculation with $d=3.42 \AA$ $[d=3.10 \AA$ ], black [gray (blue)] triangles: TB calculations with $v_{F}=1.09 \times 10^{6} \mathrm{~m} / \mathrm{s} \quad\left[v_{F}=0.79 \times 10^{6} \mathrm{~m} / \mathrm{s}\right]$. (d) Filled gray (orange) circles: Experimental data as in Fig. 1(c) compared to some calculations displayed in (c) with the same color code. The dotted (blue) line is a fit of the experimental data using Eq. (1) with $v_{F}=1.12 \times 10^{6} \mathrm{~m} / \mathrm{s}$ and $t_{\theta}=0.108 \mathrm{eV}$.

According to the expression derived from the continuum model $[9,13,18]$, the energy separation of the vHs, $\Delta E_{\mathrm{vHs}}$, follows:

$$
\Delta E_{\mathrm{vHs}}=2 \hbar v_{F} \Gamma K \sin (\theta / 2)-2 t_{\theta},
$$

where $v_{F}$ is the Fermi velocity for monolayer graphene, $\Gamma K=1.703 \AA^{-1}$ is the wave vector of the Dirac point in monolayer graphene, and $t_{\theta}$ is the modulus of the amplitude of the main Fourier components of the interlayer potential. It is thus worth checking the validity of this formula for the whole range of angles studied here, since it would then be straightforward to extract $v_{F}$ and the strength of the interlayer interaction directly from our data set. The DFT results together with two sets of TB calculations are displayed in Fig. 2(c). The TB sets differ only in the first-neighbor inplane hopping parameter to get $v_{F 1}=1.1 \times 10^{6} \mathrm{~m} / \mathrm{s}$, similar to the value of the continuum model, and a reduced $v_{F 2}=$ $0.8 \times 10^{6} \mathrm{~m} / \mathrm{s}$, similar to the one obtained by DFT. All calculations show that it is possible to recover $v_{F}$ from the slope of $\Delta E_{\mathrm{vHs}}$ vs $\sin (\theta / 2)$ as predicted by the continuum model [40]. To simulate an increase of the interlayer coupling, DFT calculations have also been performed by decreasing by $0.32 \AA$ the equilibrium interlayer distance obtained from the DFT calculation. As can be seen in Fig. 2(c), the only consequence is a rigid decrease of $\Delta E_{\mathrm{vHs}}$, which corresponds to a larger value of $t_{\theta}$, in agreement with Eq. (1). Finally, Fig. 2(d) shows that our experimental results for the $\Delta E_{\mathrm{vHs}}$ also conform to the theoretical predictions. Therefore, fitting the data of Fig. 2(d) with Eq. (1) allows us to derive an experimental value of $t_{\theta}=0.108 \mathrm{eV}$ valid in the range $1^{\circ}<\theta<10^{\circ}$ and similar to the theoretical one $[9,13,17]$ and a value $v_{F}=1.12 \times$ $10^{6} \mathrm{~m} / \mathrm{s}$, consistent with what was reported previously for monolayerlike graphene on the $\mathrm{SiC} \mathrm{C}$ face $[24,31,32,36]$.

As shown, the position of $\mathrm{vHs}$ is very sensitive to the interlayer distance, which makes crucial the incorporation of van der Waals interactions in the DFT calculations. We find the DOS to be essentially unperturbed by the spatial modulation of the interlayer distance with MP periodicity, since the DOS obtained for fully relaxed layers, and thus corrugated up to $0.1 \AA$ as shown below, is essentially the same as the one obtained when both layers are forced to remain flat [40].

We have investigated the robustness of vHs against perturbations due to subsurface stacking. In our experiments, we have taken STS data on multilayer stacks ( $>5$ layers thick) and considered only the rotation between the two uppermost surface graphene layers. Thus, we believe it is important to assess experimentally the role played by deeper layers. This can be done here by investigating domains showing multiple MP, which reveals the existence of two consecutive rotations, by small angles, between the three uppermost layers $[25,27]$. Spectra taken on multiple MP with two very different values of $\theta$ are shown in Figs. 3(a) and 3(b), respectively. In Fig. 3(a) one identifies a multiple MP with two superstructures of 1.52 and $3.7 \mathrm{~nm}$ periodicity. The former corresponds to a rotation of $\theta=9.3^{\circ}$ between the first and second graphene layers [46]. Comparing the spectrum in Fig. 3(a) to the one shown in Fig. 1(d) for a simple MP with $\theta=9.6^{\circ}$, we find that both the line shape and the vHs separation (about $1.8 \mathrm{~V}$ ) are similar. In Fig. 3(b) the multiple MP shows two periods with values 3.0 and $12.7 \mathrm{~nm}$, the latter corresponding to a rotation of $\theta=1.1^{\circ}$ between the first and second graphene layers. Local spectra reveal two peaks very close to zero bias, whose intensity strongly varies with position inside the large MP period, the same as found for the simple MP with $\theta=1.4^{\circ}$ of Fig. 1. Figure 1(e) finally shows that $\Delta E_{\mathrm{vHs}}$ in STS spectra of multiple MP (empty circles) are essentially the same as for simple MP (filled circles) when considering only the rotation $\theta$ between the two uppermost layers.

The question of the apparent corrugation of the MP in STM images is an important issue, since the corrugation of the MP has been considered as an indicator of the strength of the interlayer interaction and as an evidence for structural differences [13,36,39], which could explain the conflicting results reported for the existence of vHs in twisted multilayer samples from different origins. 


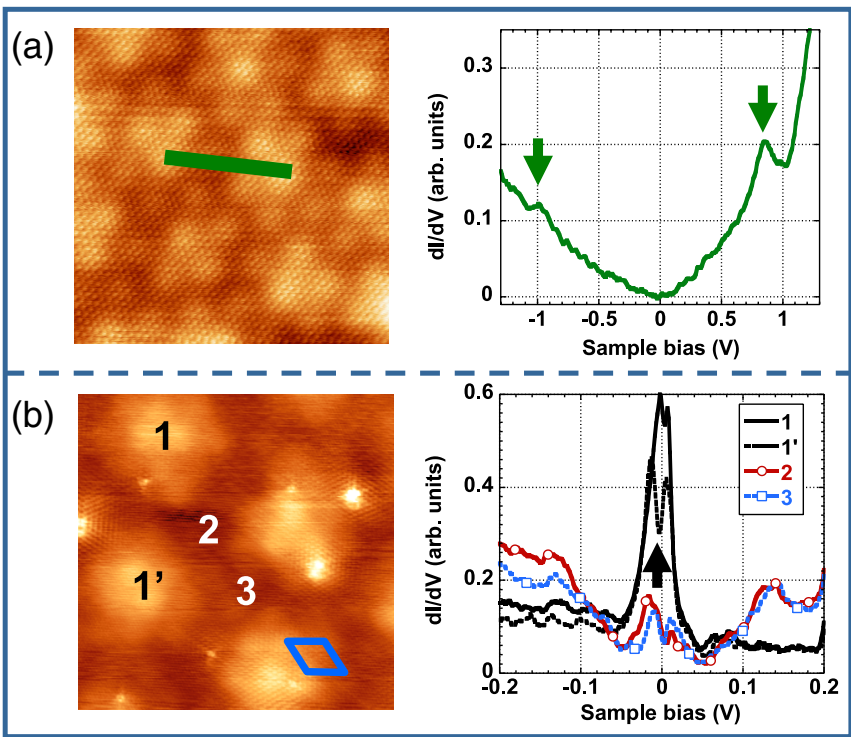

FIG. 3 (color online). (a) Left: $10.6 \times 10.6 \mathrm{~nm}^{2}$ STM image of a MP with two periods: 1.52 and $3.7 \mathrm{~nm}$. The smaller period corresponds to the MP between first and second surface layers. Right: Average spectrum taken on the green line. (b) Left: $24 \times 24 \mathrm{~nm}^{2}$ STM image of a MP with two periods: 12.7 and $3.0 \mathrm{~nm}$ (blue diamond). The larger period corresponds to the MP between first and second surface layers. Right: Local spectra taken on the spots labeled on the image. Arrows point to the vHs.

Figures 4(a) and 4(b) show representative STM images for two simple MP corresponding to $6.1^{\circ}$ and $3.3^{\circ}$ rotation angles, respectively, which then present very different corrugations. The MP corrugation, defined here as the height difference between the highest and lowest areas along the MP axis, has been measured on more than 20 different MP, using different tips, tunneling impedances (from $80 \mathrm{M} \Omega$ to $5 \mathrm{G} \Omega$ ), and sample biases (from $10 \mathrm{mV}$ to $1 \mathrm{~V})$. For a given MP, the measured corrugation depends on the tip state and tunneling parameters; only the maximum and minimum values of the measured corrugation were included to construct the graphs in Fig. 4(c). It appears immediately that the corrugation tends to increase with decreasing angle and that the contrast varies considerably (up to a factor of 3 ) with the experimental conditions for low angles $\left(<4^{\circ}\right)$. This gives evidence for a significant electronic effect in the contrast of large MP as previously reported $[26,27,47,48]$.

Comparison with DFT computed corrugations allows a quantitative estimate of the relative importance of topographic and electronic effects in the apparent contrast found by STM. Figure 4(d) shows the theoretical STM image for a MP with $6.01^{\circ}$. Ab initio calculation reproduces quite nicely the experimental MP corrugation measured for the $\theta=6.1^{\circ}$ MP of Fig. 4(a) (similar agreement is obtained for other angles). The bright areas correspond to a local $A A$ stacking, as quoted previously [26,47,48]. As shown in Fig. 4(e), where the interlayer distance (dots) and the total computed

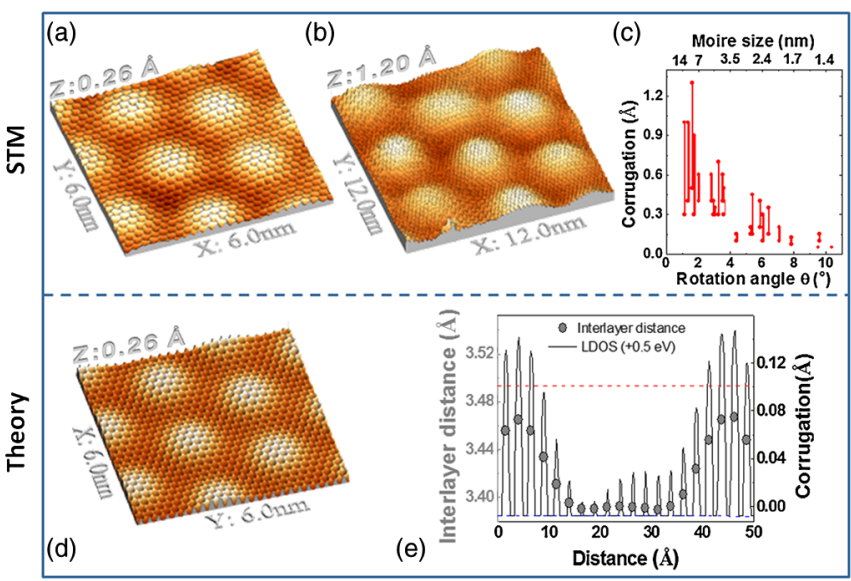

FIG. 4 (color online). (a) STM image at sample bias $V=$ $+0.5 \mathrm{~V}$ of a MP with $P=2.32 \mathrm{~nm}\left(\theta=6.08^{\circ}\right)$. The measured corrugation is $0.14 \AA$. (b) STM image at $V=+0.2 \mathrm{~V}$ of a MP with period $P=4.3 \mathrm{~nm}\left(\theta=3.28^{\circ}\right)$. (c) Range of STM measured MP corrugation as a function of rotation angle $\theta$. (d) Simulated STM image for a bilayer rotated $\theta=6.01^{\circ}$ at $+0.5 \mathrm{eV}$. (e) Atomic (dots) and total (line) corrugation for the bilayer image shown in (d) along the long MP diagonal. The blue and red horizontal dashed lines indicate the interlayer spacing computed for $A B$ and $A A$ bilayers, respectively.

STM corrugation (lines) are plotted, only half of the total corrugation $(0.14 \AA)$ is of topographic origin $(0.075 \AA)$. Interestingly, for this MP, the interlayer distance varies between values close to those computed for infinite $A A$ and $A B$ graphene bilayers [red and blue lines in Fig. 4(e)]. Thus, although the regions with, e.g., $A A$-like stacking are quite small for this $\theta=6.01^{\circ}$ value, typically $7 \mathrm{C}$ hexagons in size [47], the interaction between planes is sufficient as to set the interlayer distance close to the value found for the uniform $A A$ stacked layers. Thus, despite the huge apparent corrugation measured by STM in large MP, our calculations set the upper limit to the actual topographic corrugation of twisted graphene layers to be $0.11 \AA$ [40], the difference in interlayer distance between the uniform $A A(3.495 \AA)$ and $A B$ (3.384 ̊̊) stacked phases.

Our results conclusively demonstrate that the LDOS of two graphene layers stacked with rotation angles between $1^{\circ}$ and $10^{\circ}$ exhibit logarithmic vHs. Extensive STS data allow the determination of the effective interlayer coupling parameter $t_{\theta} \approx 0.11 \mathrm{eV}$. Numerical simulations and experiments indicate that these $\mathrm{vHs}$ are robust against perturbations in the interlayer distance and in the stacking of the underlying graphene layers. We also analyze the origin of the contrast of the moire pattern in STM images, discriminating the contributions of the layer corrugation and of the electronic effects.

We thank J. M. Soler, F. Hiebel, D. Mayou, and V. Olévano for fruitful discussions. This work was supported by Spain's MICINN under Grants No. MAT2010-14902, No. CSD2010-00024, and No. CSD2007-00050, and by Comunidad de Madrid under Grant No. S2009/MAT-1467. 
M. M. U., I. B., P. M, J.-Y. V., L.M., and J.M. G.-R. also acknowledge the PHC Picasso program for financial support (Project No. 22885NH). I. B. was supported by a Ramón y Cajal project of the Spanish MEC. L. M., P. M., and J.-Y. V. acknowledge support from Fondation Nanosciences (Dispograph project).

*jean-yves.veuillen@grenoble.cnrs.fr

[1] S. Novoselov, A. K. Geim, S. V. Morozov, D. Jiang, M. I. Katsnelson, I. V. Grigorieva, S. V. Dubonos, and A. A. Firsov, Nature (London) 438, 197 (2005).

[2] Yuanbo Zhang, Yan-Wen Tan, Horst L. Stormer, and Philip Kim, Nature (London) 438, 201 (2005).

[3] Claire Berger et al., Science 312, 1191 (2006).

[4] C.-H. Park, L. Yang, Y.-W. Son, M. L. Cohen, and S. G. Louie, Nat. Phys. 4, 213 (2008).

[5] C.-H. Park, L. Yang, Y.-W. Son, M. L. Cohen, and S. G. Louie, Phys. Rev. Lett. 101, 126804 (2008).

[6] S. Rusponi, M. Papagno, P. Moras, S. Vlaic, M. Etzkorn, P. M. Sheverdyaeva, D. Pacile, H. Brune, and C. Carbone, Phys. Rev. Lett. 105, 246803 (2010).

[7] J. Sanchez-Barriga, A. Varykhalov, D. Marchenko, M. R. Scholz, and O. Rader, Phys. Rev. B 85, 201413(R) (2012).

[8] M. Yankowitz, J. Xue, D. Cormode, J. D. Sanchez-Yamagishi, K. Watanabe, T. Taniguchi, P. Jarillo-Herrero, P. Jacquod, and B. J. LeRoy, Nat. Phys. 8, 382 (2012).

[9] J. M. B. Lopes dos Santos, N. M. R. Peres, and A. H. Castro Neto, Phys. Rev. Lett. 99, 256802 (2007).

[10] S. Latil, V. Meunier, and L. Henrard, Phys. Rev. B 76, 201402(R) (2007).

[11] J. Hass, F. Varchon, J. E. Millán-Otoya, M. Sprinkle, N. Sharma, W. A. de Heer, C. Berger, P. N. First, L. Magaud, and E. H. Conrad, Phys. Rev. Lett. 100, 125504 (2008).

[12] S. Shallcross, S. Sharma, and O. A. Pankratov, Phys. Rev. Lett. 101, 056803 (2008).

[13] G. Li, A. Luican, J. M. B. Lopez dos Santos, A. H. Castro Neto, A. Reina, J. Kong, and E. Andrei, Nat. Phys. 6, 109 (2010).

[14] G. Trambly de Laissardière, D. Mayou, and L. Magaud, Nano Lett. 10, 804 (2010).

[15] E. Suarez Morell, J. D. Correa, P. Vargas, M. Pacheco, and Z. Barticevic, Phys. Rev. B 82, 121407(R) (2010).

[16] S. Shallcross, S. Sharma, E. Kandelaki, and O. A. Pankratov, Phys. Rev. B 81, 165105 (2010).

[17] G. Trambly de Laissardière, D. Mayou, and L. Magaud, Phys. Rev. B 86, 125413 (2012).

[18] J. M. B. Lopes dos Santos, N. M. R. Peres, and A. H. Castro Neto, arXiv:1202.1088.

[19] R. Bistritzer and A. H. MacDonald, Proc. Natl. Acad. Sci. U.S.A. 108, 12233 (2011).

[20] P. San-Jose, J. Gonzalez, and F. Guinea, Phys. Rev. Lett. 108, 216802 (2012).

[21] Y. Murata, S. Nie, A. Ebnonnasir, E. Starodub, B. B. Kappes, K. F. McCarty, C. V. Ciobanu, and S. Kodambaka, Phys. Rev. B 85, 205443 (2012).

[22] B. Fallahazad, Y. Hao, K. Lee, S. Kim, R. S. Ruoff, and E. Tutuc, Phys. Rev. B 85, 201408(R) (2012).

[23] F. Varchon, P. Mallet, L. Magaud, and J-Y Veuillen, Phys. Rev. B 77, 165415 (2008).
[24] D. L. Miller, K. D. Kubista, G. M. Rutter, M. Ruan, W. A. de Heer, P. N. First, and J. A. Stroscio, Science 324, 924 (2009).

[25] D. L. Miller, K. D. Kubista, G. M. Rutter, M. Ruan, W. A. de Heer, P. N. First, and J. A. Stroscio, Phys. Rev. B 81, 125427 (2010).

[26] Z. Y. Rong and P. Kuiper, Phys. Rev. B 48, 17427 (1993).

[27] W.-T. Pong and C. Durkan, J. Phys. D 38, R329 (2005).

[28] T. Ohta, T.E. Beechem, J. T. Robinson, and G. L. Kellogg, Phys. Rev. B 85, 075415 (2012).

[29] R. W. Havener, H. Zhuang, L. Brown, R. G. Henning, and J. Park, Nano Lett. 12, 3162 (2012).

[30] K. Kim, S. Coh, L.Z. Tan, W. Regan, J. M. Yuk, E. Chatterjee, M. F. Crommie, M. L. Cohen, S. G. Louie, and A. Zettl, Phys. Rev. Lett. 108, 246103 (2012).

[31] M. L. Sadowski, G. Martinez, M. Potemski, C. Berger, and W. A. de Heer, Phys. Rev. Lett. 97, 266405 (2006).

[32] M. Sprinkle et al., Phys. Rev. Lett. 103, 226803 (2009).

[33] W. A de Heer et al., J. Phys. D 43, 374007 (2010).

[34] M Sprinkle et al., J. Phys. D 43, 374006 (2010).

[35] A. Luican, Guohong Li, A. Reina, J. Kong, R. R. Nair, K. S. Novoselov, A. K. Geim, and E. Y. Andrei, Phys. Rev. Lett. 106, 126802 (2011).

[36] J. Hicks et al., Phys. Rev. B 83, 205403 (2011).

[37] Dong Su Lee, C. Riedl, T. Beringer, A. H. Castro Neto, K. von Klitzing, U. Starke, and J. H. Smet, Phys. Rev. Lett. 107, 216602 (2011); J. D. Sanchez-Yamagishi, T. Taychatanapat, K. Watanabe, T. Taniguchi, A. Yacoby, and P. Jarillo-Herrero, Phys. Rev. Lett. 108, 076601 (2012).

[38] R. Bistritzer and A.H. MacDonald, Phys. Rev. B 84, 035440 (2011); Pilkyung Moon and Mikito Koshino, Phys. Rev. B 85, 195458 (2012).

[39] E. J. Mele, Phys. Rev. B 84, 235439 (2011).

[40] See Supplemental Material at http://link.aps.org/ supplemental/10.1103/PhysRevLett.109.196802 for details on $a b$ initio calculations and additional STM and STS data.

[41] M. M. Ugeda, I. Brihuega, F. Guinea, and J. M. GomezRodriguez, Phys. Rev. Lett. 104, 096804 (2010).

[42] I. Brihuega, P. Mallet, C. Bena, S. Bose, C. Michaelis, L. Vitali, F. Varchon, L. Magaud, K. Kern, and J. Y. Veuillen, Phys. Rev. Lett. 101, 206802 (2008).

[43] M. M. Ugeda, I. Brihuega, F. Hiebel, P. Mallet, J-Y. Veuillen, J. M. Gomez-Rodriguez, and F. Yndurain, Phys. Rev. B 85, 121402(R) (2012).

[44] I. Horcas, R. Fernandez, J. M. Gomez-Rodriguez, J. Colchero, J. Gomez-Herrero, and A. M. Baro, Rev. Sci. Instrum. 78, 013705 (2007).

[45] L.Z. Kong, G. Roman-Perez, J. M. Soler, and D. C. Langreth, Phys. Rev. Lett. 103, 096103 (2009); M. Dion, H. Rydberg, E. Schroder, D. C. Langreth, and B. I. Lundqvist, Phys. Rev. Lett. 92, 246401 (2004).

[46] The stacking sequence has been deduced from the angle between the surface graphene lattice and the MP $[23,25,27]$ : for a rotation angle $\theta$ between twisted layers the surface graphene lattice should be rotated by $30^{\circ}-(\theta / 2)$ relative to the main axis of the MP. This is the case in Fig. 3(a) for the small period MP with $\theta=9.3^{\circ}$, but not for the large MP. The same argument holds for the MP in Fig. 3(b).

[47] J. M. Campanera, G. Savini, I. Suarez-Martinez, and M. I. Heggie, Phys. Rev. B 75, 235449 (2007).

[48] E. Cisternas and J. D. Correa, arXiv:1202.2612. 